\title{
Development of a network level crossing system
}

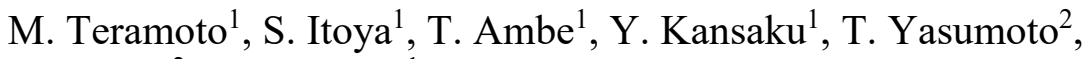 \\ Y. Konno ${ }^{2}$ \& Y. Fukuta ${ }^{1}$ \\ ${ }^{1}$ East Japan Railway Company, Japan \\ ${ }^{2}$ Toshiba Corporation, Japan
}

\begin{abstract}
A level crossing which is a cross point between an automobile road and a railroad is unavoidable for a railway operator. Of course, the safety at a level crossing is top priority and, at the same time, we have to consider the convenience and availability for passers by (pedestrians, vehicles, etc.).

Basically, a current level crossing controller is a standalone device. Once it detects a fault, such as lightning stroke damage to a train sensor, it makes/keeps warning according to the fail-safe philosophy and stops passers by entering the train area.

However, this long time warning causes not only inconvenience for the passer by but also dangerous situations. For example, it may interrupt emergency vehicles, or it may cause situations where a frustrated passer by would run through the level crossing barriers and enter the train area.

Recent ICT (information communication technology) progress has been applied to solve our issues. We have developed a network level crossing system. More than three level crossing controllers are connected through the Ethernet LAN connection to exchange their operational data. At normal state, they are operating as stand-alone machines, once one controller detects a problem, it operates not by means of its own data but by another controller's data. As a result, by a degraded level crossing function, passers by need not undergo unnecessary extensive warning.

We have produced the network level crossing system experimentally and examined it in house. Numerical estimation using actual train operation data shows that in cases of malfunction of a train detector, the current level crossing system warns every time, whereas our system warns only $18.7 \%$ of the time. It means that the current system completely blocks passers by and our system provides many chances to pass the level crossing. Our system achieves the convenience and
\end{abstract}


availability of level crossings. In addition, it improves the safety of level crossings. Furthermore, it is expected to achieve the safety and convenience of level crossings as a next generation level crossing system.

Keywords: level crossing, long time warning, network, Ethernet, microelectronic level crossing controller.

\section{Introduction}

In Japan, there are many level crossings, which are weak points for railroad operations. The safety of a level crossing is top priority. In addition, the long warning time of level crossing has been a social problem recently [1,2]. The traffic of passers by (pedestrians, vehicles, etc.) is disturbed for a long time thus we have to consider the convenience for them.

A crossing with an overpass or underpass is the final goal to eliminate a level crossing, but it needs so much time and cost unfortunately. Therefore, a level crossing system performing adequate warning is required as a practical solution.

An extraordinary long time warning is mainly caused by the following two problems. One problem is that since not all level crossings have achieved their optimized control, warning times are longer than those of theoretically minimum ones in many cases. In particular, on the train line where many types of trains run, a low-speed train causes long time warning because the starting point of level crossing warning is geographically arranged according to the fastest train of the line.

The other problem is that a level crossing controller ceases control and makes/keeps warning in order to ensure a safe state (to avoid the passers by entering into the train area) when it detects an error and until it is repaired.

A constant warning time control is a solution for the first problem [3]. It has been widely adopted at JR East as countermeasures against level crossing barrier closing time elongation. Its control logic is realized by changing the starting point according to train types.

On the other hand, to solve the second problem we have to totally eliminate problems around a level crossing but it is not easy. Recently, many systems using ICT (Information Communication Technology) have been developed. For instance, the railway signal system which controls devices by using the internet protocol to transmit the signal command not via a metal cable but via an optical Ethernet cable was developed and installed $[4,5]$. Moreover, train control systems using radios have been realized $[6,7]$ and a system, named "ATACS" operates commercially in Japan [8]. ICT has great potential to change the current signalling systems, so we have improved a level crossing system using ICT.

\section{Schematics of a network level crossing system}

\subsection{Current level crossing systems}

Usually a level crossing is a stand-alone system even where several level crossings are closely arranged. Figure 1 shows a level crossing system configuration 
between stations; Level crossing B, C, D. As the control of an individual level crossing is independent, each level crossing operates in the assigned area. A typical system consists of a starting point (detector $\mathrm{C} 1$ when we look at the level crossing $\mathrm{C}$ in Fig. 1), a stopping point (detector $\mathrm{C} 2$ ) and a microelectronic controller. The level crossing controller counts the number of trains detected by $\mathrm{C} 1$ and $\mathrm{C} 2$ then controls warnings.

Once a level crossing detects a failure, it constantly emits a warning and blocks traffic to keep a safe state to avoid passers by entering into the train running area. In such a level crossing failure, maintenance personnel go to the site and restore the level crossing after investigating the cause, so the warning and traffic blocking continues for a long time. Particularly when such a failure occurs at a location such as a level crossing on a main street with much traffic, this has a major social impact.

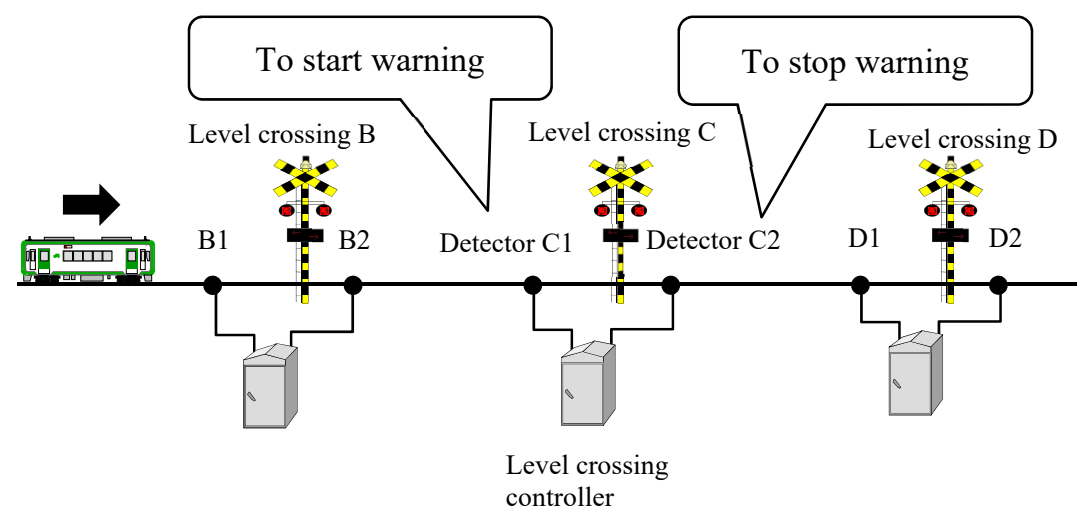

Figure 1: System configuration of level crossing.

\subsection{Basis of our network level crossing system}

Taking these issues into account, we have developed a level crossing control system where adjacent microelectronic level crossings are connected to each other over a network. Figure 2 schematically illustrates the network level crossing system and its function. More than three crossings are connected through the network in order to exchange operational data. Usually, they are operating as stand-alone machines. Once one controller detects a problem, it operates not by means of its own data but another controller's data. The following shows the case when detector $\mathrm{C} 1$ is damaged. When the controller of level crossing $\mathrm{C}$ detects a failure of the detector $\mathrm{C} 1$, the level crossing $\mathrm{C}$ does not use the data of itself but uses the data of level crossing B and level crossing D. Thus, the control area of the degraded system shifts to the area covering level crossing B to level crossing D. 


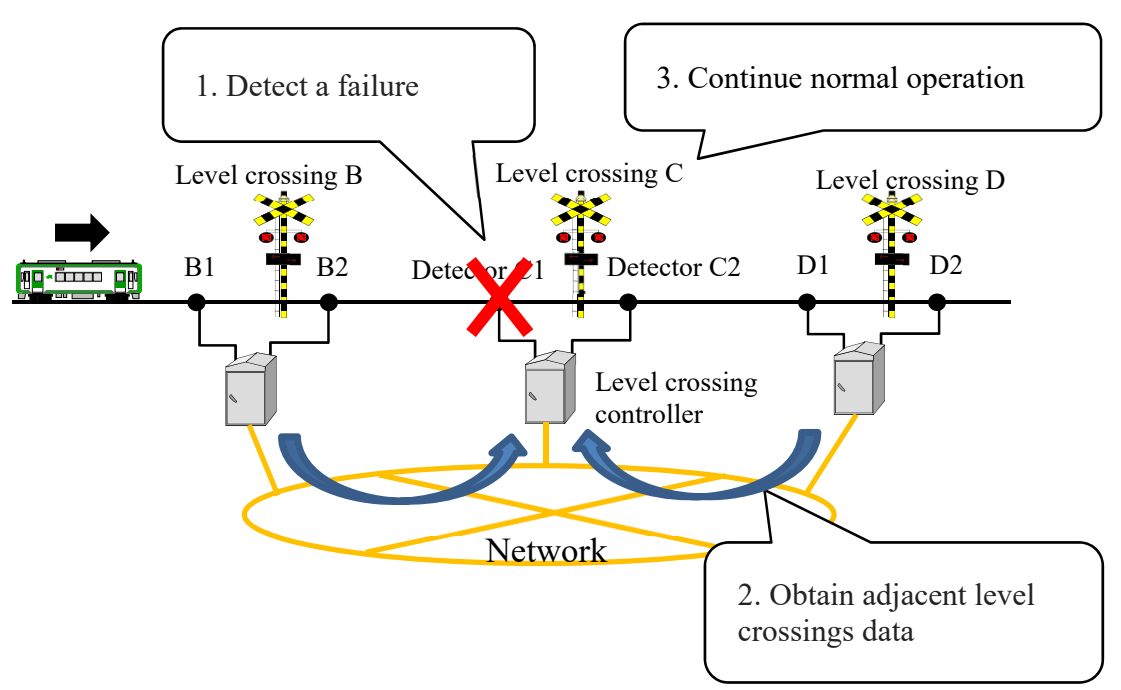

Figure 2: Illustration of the network level crossing system.

\section{The control logic of a network level crossing system}

\subsection{Managing the train location}

A conventional level crossing checks train locations using a train number counter that indicates the number of trains in the control area of the level crossing. In the case of level crossing $\mathrm{C}$ in Figs 2 or 3, the detector $\mathrm{C} 1$ increments the train number counter and the detector $\mathrm{C} 2$ decrement it. However, once a failure occurs such as if the train number counter becomes unreliable, the level crossing has to cease control based on the train counter and a full block of level crossing is needed because we cannot know when a train passes.

In order to overcome this problem, we have developed a new level crossing controller which has two train counters. One is the same counter as a current level crossing controller and the other is a new counter named approaching train number counter which can manage the number of trains running in the area between adjacent level crossings on both sides. To realize this counter, our level crossing always exchanges the number of trains running its control area.

The number of trains in the approaching train number counter increases or decreases according to changes of the train number of level crossing B and D. Moreover, when a level crossing detects that it has failed, the system changes into a degraded mode using the approaching train counter. In this way, train location 
control using the approaching train number counter can ensure the same safety level as the conventional train location management. Figure 3 shows the concept image.

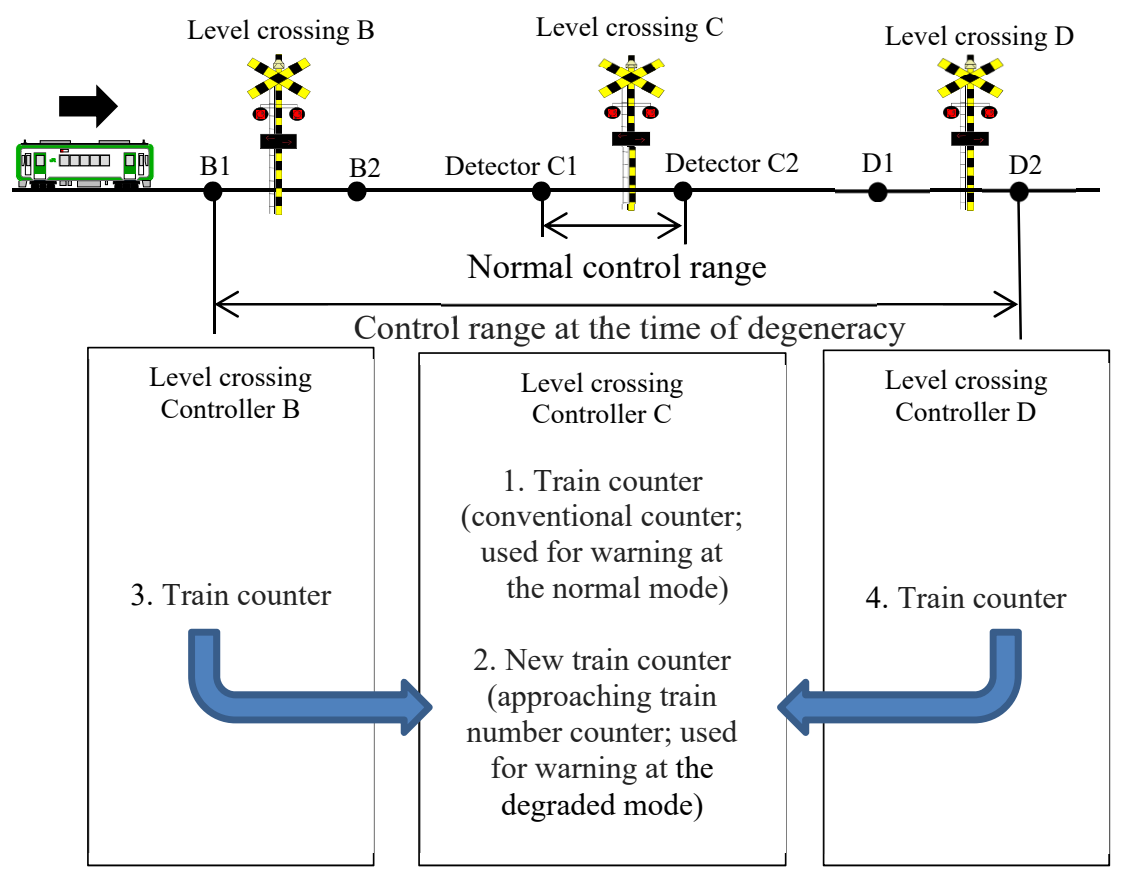

Figure 3: Conceptual diagram of network level crossing.

We have proposed to control level crossings using information of adjacent level crossings, but it is not desirable to develop a completely new logic. We thus made full use of the logic of adjacent level crossings that are fail-safe devices. Specifically, the level crossing network system relies on adjacent level crossings detecting failures by themselves and changes to degraded mode only when the adjacent level crossings have not failed. In this way, level crossing $\mathrm{C}$ does not need to judge the precise conditions of level crossings B and D.

\subsection{Four status of a network level crossing system}

For a safe control, a level crossing requires an appropriate control method according to the state of the moment. The level crossing controller has four modes in accordance with status of itself and the network (the controller is normal/abnormal state and normal/abnormal obtaining of data of adjacent level crossings via the network).

The following illustrates the four modes. 
(a) Network Mode (Level crossing controller is normal and Network is normal) Both a level crossing and the network are in normal status. At this mode, the level crossing controller is operating as a stand-alone device, and constantly obtains information of the adjacent level crossings to operate the degraded logics in the background to assure safety even while the system is operating normally. We defined this status as Network Mode.

(b) Degraded Mode (Level crossing controller is abnormal and Network is normal) This is the status after the level crossing concerned detects a failure and the system switches to degraded mode. In this mode, using the approaching train number counter as explained in Fig. 3, the system assures safe control by performing train location management the same as with conventional control. We defined this status as Degraded Mode.

(c) Local Mode (Level crossing controller is normal and Network is abnormal) This is the status when a level crossing operates normally but it cannot obtain information from the adjacent level crossings or information is unreliable because of a failure at the network. In addition to the conventional functions, we designed our system not to perform incorrect mode transition that may result in a dangerous situation. Thus we assured the safety level equivalent to that of conventional control. We defined this status as Local Mode.

(d) Failure Mode (Level crossing controller is abnormal and Network is abnormal) This is the status when both a level crossing and the network are in an abnormal state. In this case, safety is not assured because calculation results of a standalone level crossing are unreliable as well as those calculated using the information of adjacent crossings. When the system finds this situation, i.e. detects the abnormal state of controller itself and unreliable state of the network, it makes/keeps warning to prevent dangerous results. We defined this status as Failure Mode.

As mentioned above, we defined four control modes to secure safety and investigated individual transition conditions. Figure 4 shows the mode transition chart. The startup process firstly runs when the power switch is turned on and selfdiagnosis runs simultaneously. Secondly, with good results of the startup process, the system shifts to Local Mode. Thirdly, after a health check of the network, it shifts to Network Mode that is expected as the usual status of the system and the level crossings. In the case of trouble or failure around the level crossing or the network, the system detects a failure part and changes its mode to the corresponding one. 


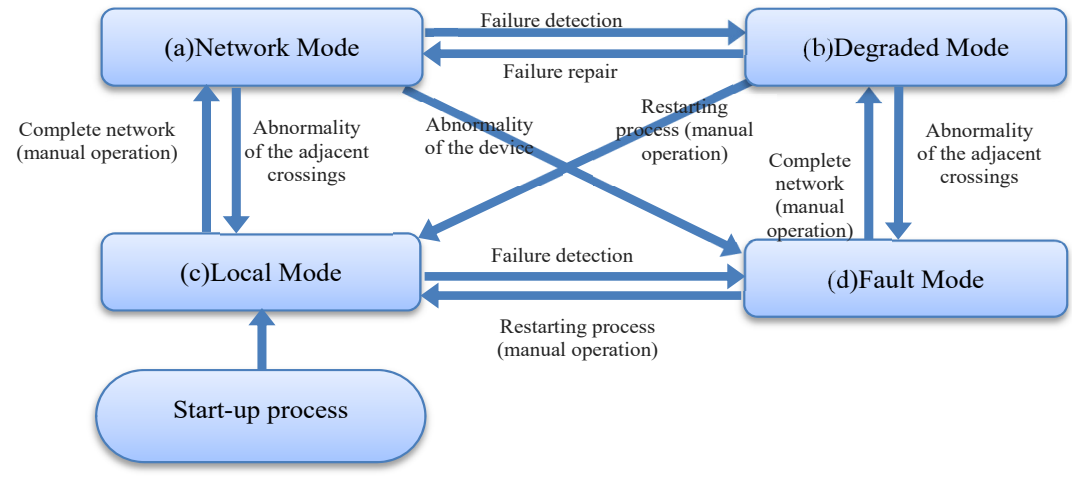

Figure 4: The state transition of the four modes.

\section{Evaluation of the system}

We have experimentally produced a setup of the network level crossing system and examined it in house. Configuration of the setup is shown in Figure 5.

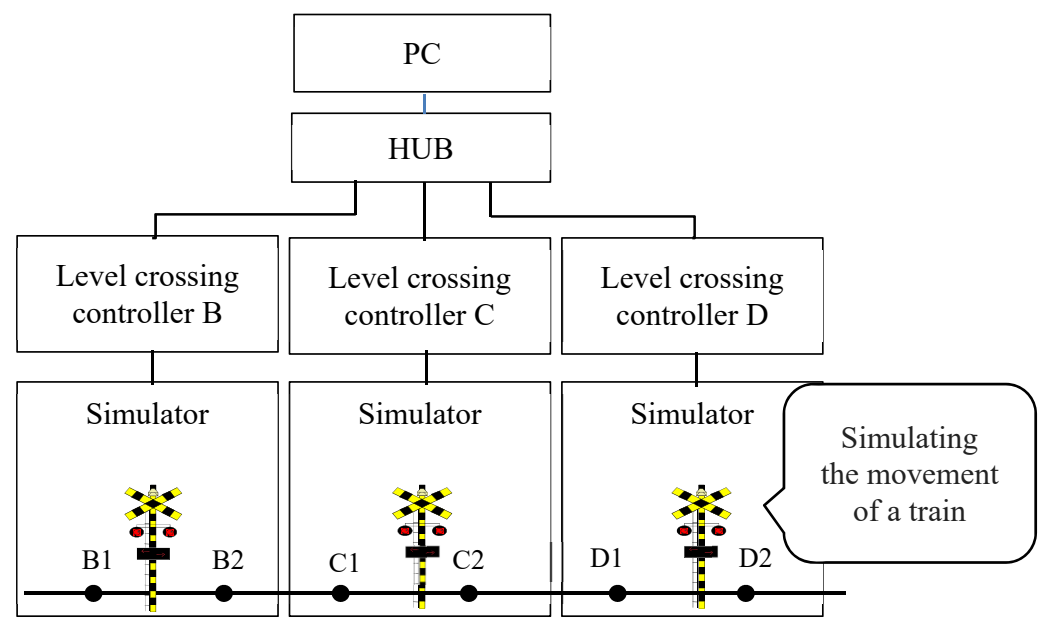

Figure 5: System configuration of the evaluation setup. 
Figure 5 shows the conceptual configuration and Figure 6 shows a photo of testing in progress. In the system configurations, three prototype microelectronic level crossings were placed in a row and connected to each other by Ethernet via a hub. The PCs connected to the hub were provided with a remote monitoring function developed to confirm failures of the microelectronic level crossing remotely and a monitoring terminal developed to monitor the internal condition of the level crossing system. Moreover, in order to simulate the operation of the onsite controller and check the output power of the level crossings on a LED display, a switch panel was produced as well.

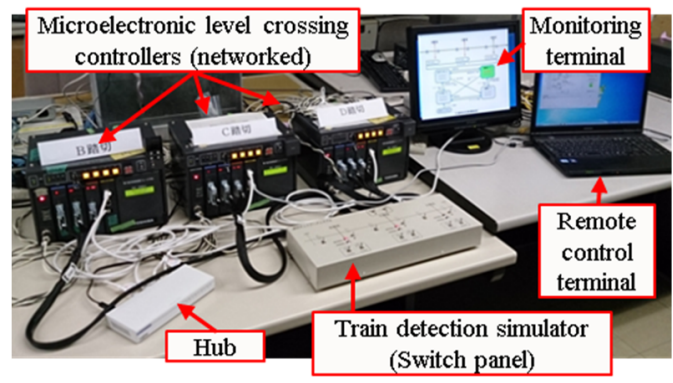

Figure 6: Function check test setup.

We carried out tests such as input/output tests, network tests, approaching train number count tests, control functions tests in the modes of introduced above, and all functions of the system operated without problem as defined in the specifications.

\section{Effect of a network level crossing system}

We estimated the effect of the network level crossing system. Assuming a case where the system operates in degraded mode in a section with many betweenstation level crossings in the Tokyo Suburban area in the time slot from 7:00 to 7:59 on a weekday. Table 1 shows the results of the estimation. When a conventional level crossing detects a failure, it constantly emits warning and blocks traffic, so the rate of warning in one hour is $100 \%$. In contrast with the level crossing network system operating in degraded mode, that rate is $18.7 \%$, showing that it achieves a warning time shorter than that of conventional level crossings.

Table 1: Warning time per hour at a level crossing.

\begin{tabular}{|c|c|c|}
\hline & Normal & $\begin{array}{c}\text { Failure detected } \\
\text { (on outbound line) }\end{array}$ \\
\hline Current level crossing & $8.4 \min (14 \%)$ & $60 \min (100 \%)^{*}$ \\
\hline $\begin{array}{c}\text { Network level crossing } \\
\text { system (developed) }\end{array}$ & $8.4 \min (14 \%)$ & $11.2 \min (18.7 \%)$ \\
\hline
\end{tabular}

* keep warning until repaired 


\section{Future work}

We have mainly studied the effect of a level crossing on a double track line. We have also investigated the effect on a single track line. Within a desk study, our system has approximately fulfilled the demands of a single track line, but further investigation will be undertaken.

In this study, the LAN network was experimentally applied as a communication network among level crossings, but high safety and reliability of the communication network are required in actual rail. The new signaling system where the normal internet protocol is used to control signal devices [4, 5] will solve this problem. Furthermore, application of wireless sensor network technology to railway signaling system [9] will be useful for the network. Currently the application for monitoring rail signaling devices are developed [10] and further development is expected.

\section{Conclusion}

We have developed a new level crossing system that enables to reduce influence of a level crossing failure and improves the conveniences and availability for passers by. Thanks to recent progress of ICT, the network technology has been applied to connect electronic level crossing controllers and cooperating functions of the controllers have been realised. Today, in the case of a failure of a level crossing, the crossing has to make/keep warning in order to assure safety. This is because the conventional level crossing controller is a standalone device, so once the information for control is found to be unreliable, the controller ignores it and blocks passers by from entering the train running area. This is based on a fail-safe philosophy, but it decreases convenience and availability for passers by at the same time.

For our network level crossing system, four modes are essential, which are determined by the failure parts; Network Mode, Degraded Mode, Local Mode and Failure Mode. They are proposed and investigated in this study, among which the level crossing controller shifts in order to achieve proper controls.

We have produced an experimental prototype system and examined it in house. The expected functions have been practically realized. Moreover, numerical estimation using actual train operation data was also examined. In the case of malfunction of a train detector, the current level crossing system warns all the time, whereas our system warns only $18.7 \%$ of the time. It means that the current system completely blocks the passer by and our system provides many opportunities to pass the level crossing. Our system achieves convenience and availability of level crossings.

Further developments are expected. At first we have to expand the application field. As far as a level crossing between stations on a double track line is concerned, almost all situations are examined in order not to cause an unsafe situation. The next step is expanding to a level crossing on a single track line. Our desk study roughly shows that our system is available for a single track line, but detailed investigation is required. 
In addition, recent progress of ICT must improve our system. For example, sensor network technology or radio communication technology will be very useful to our system. Using these excellent technologies, our system will realize the safety and conveniences of a level crossing as a next generation level crossing system.

\section{References}

[1] Ian McCullough, "International Barriers to Level Crossing", IRSE-ITC on behalf of the International Technical Committee of the IRSE. IRSE-ITC report, http://www.irse.org/knowledge/ public/itc.aspx

[2] Yuji Hirao, "Improving Safety at Level Crossings, Japanese Developments", Paper delivered to UIC International Railway Research Board Meeting, November, 21, Paris, France, 2005.

[3] Manabu Teramoto, et al., "Standardization of logic for a constant warning time control at automatic level crossings", Proceedings of the 14th International Conference on Railway Engineering Design and Optimisation (COMPRAIL 2014), June 24-26, Rome, Italy, 2014.

[4] Yoshiyuki Hirano, et al., "Development of Railway Signaling System based on Network Technology", Proceedings of IEEE International Conference on Systems, Man \& Cybernetics (IEEE SMC2005), October 10-12, Hawaii, USA, 2005.

[5] Yamato Fukuta, et al., "Novel Railway Signal Control System Based on the Internet Technology and Its Distributed Control Architecture" Proceedings of The 8th International Symposium on Autonomous Decentralized Systems (IEEE ISADS 2007), March 21-23, Sedona, USA, 2007.

[6] The European Rail Traffic Management System (ERTMS), http://www.ertms.net/

[7] Communications-based Train Control (CBTC), https://standards.ieee.org/ findstds/standard/1474.1-2004.html

[8] Yuichi Baba, et al., "Train Control System based on Radio (ATACS)", JR EAST Technical Review, No.05, Autumn, pp31-38, 2003. (in Japanese) http://www.jreast.co.jp/development/tech/pdf_5/31-38.pdf

[9] Yamato Fukuta, "Possibility of Sensor Network Applying for Railway Signal System" Proceedings of Fifth International Conference on Networked Sensing Systems (INSS2008), June 17-19, Kanazawa, Japan, 2008.

[10] Masahiko Suzuki and Takashi Kato, "The Monitoring System for Railway Signal Equipment using Radio Communication - Development for Motor Point Machine and Crossing Control System" - Proceedings of Railway Engineering 2015 - 13th International Conference \& Exhibition, June 30July 1, Edinburgh, UK, 2015. 